\title{
Simulation of the Fatigue Behaviour of a Power Plant Steel with a Damage Variable
}

\author{
Jiawa Lu ${ }^{1 *}$, Wei Sun ${ }^{1}$, Adib Becker ${ }^{1}$, Abdullah Aziz Saad ${ }^{2}$ \\ ${ }^{1}$ Faculty of Engineering, University of Nottingham, Nottingham, NG7 2RD, UK \\ ${ }^{2}$ School of Mechanical Engineering, Universiti Sains Malaysia \\ *Corresponding Author Email: eaxj12@nottingham.ac.uk
}

\begin{abstract}
The fatigue behaviour of a 9Cr power plant steel at a temperature of $600{ }^{\circ} \mathrm{C}$ was studied by uniaxial fatigue tests and microstructural analysis using electron microscopy. A continuum damage mechanics apporach was coupled to the constitutive equations of the Chaboche elasto-visco-plastic model to describe the fatigue behaviour of the 9Cr power plant steel at $600{ }^{\circ} \mathrm{C}$. A stress partition method is introduced to understand the fatigue softening behaviour, and used to give an initial estimate of the material constants in the Chaboche model. Further optimisation procedures with plastic strain range dependency of the material constants were introduced in the optimisation procedure in order to accurately predict the material behaviour, especially after damage initiation. An ABAQUS UMAT subroutine was coded to allow the full life cyclic softening behaviour of a power plant component to be accurately predicted in a finite element calculation. The multiaxial capability of the coding is validated against notched bar test data.
\end{abstract}

Keywords: Cyclic plasticity; Chaboche visco-plasticity model; Continuum damage mechanics; UMAT subroutine

\begin{tabular}{|llllll|}
\hline Nomenclature & & & & \\
$\boldsymbol{\sigma}$ & Stress tensor & $D$ & Damage & $f y$ & yield function \\
$\boldsymbol{\varepsilon}$ & Strain tensor & $\Delta$ & Range & $p$ & Accumulated plastic strain \\
$\boldsymbol{X}$ & Kinematic hardening tensor & & $R$ & Isotropic hardening \\
$\boldsymbol{n}$ & Normal direction & $E$ & Young's modulus & $N_{f}$ & Number of cycles to failure \\
$\widetilde{\boldsymbol{\sigma}}$ & Effective stress tensor & $\sigma_{y}$ & Yield stress & $L$ & Life portion \\
$\boldsymbol{I}$ & Identity matrix & $t$ & Time & $\sigma_{v}$ & Viscous stress \\
$\tilde{\tilde{J}} \boldsymbol{e}$ & Effective elastic Jacobian matrix & $U$ & Displacement \\
$G$ & Shear Modulus & $\rho$ & Dislocation density & $v$ & Poisson's ratio \\
\hline
\end{tabular}




\section{Introduction}

Martensitic steel P91 is widely used in power generation plants for steam piping systems, which may be subject to long term steady and cyclic loading conditions at high temperatures. Due to the intermittent nature of renewable energy generation, conventional power plants are now subject to a higher frequency of thermo-mechanical cycling, demanded by 'flexible' operation. Due to this reason, the study of visco-plasticity behaviour of advanced power plant steels has become increasingly important. The aim of this study is to simulate the full life low cycle fatigue behaviour of a P91 steel at high temperature, and to investigate the physical evidence behind the stress and strain evolution.

Plastic and creep deformation were analysed separately historically. Starting from 1970, the flow rules for plastic and creep deformations were combined, and plasticity formulations were considered as special cases of viscoplasticity formulations [1]. A continuum approach was usually used in such analyses, which assumes that heterogeneous behaviour can be averaged through a homogeneous representative volume element (RVE), and the size of RVE for metals is normally at the order of $0.1 \mathrm{~mm}^{3}$ [2]. Chaboche and Rousselier [3] and Chaboche [4] included isotropic and kinematic hardening in a visco-plasticity flow rule, to take the cyclic hardening and the Bauschinger effect into account.

The Chaboche elasto-visco-plasticity model has been used extensively to study the cyclic material behaviour for P91. Ishmurzin et.al. [5] used this model in a typical forging problem to predict the residual stress and to estimate the fatigue life time. Saad et.al. [6] used this model to predict the initial stress amplitude evolution under strain-controlled uniaxial fatigue tests at high temperature. However, it is also important to predict the component lifetime as well as the failure site. Cracks and voids would significantly influence the cyclic stress-strain response. Following on the previous studies [6, 7], the Chaboche model was employed in this study to investigate the cyclic visco-plasticity behaviour in the full cycle fatigue life.

Historically, the scalar damage parameter $D(0 \leq D \leq 1)$ was first introduced by Kachanov [8] and modified by Rabotnov [9], to describe the fraction of the actual load bearing area over the original area. Damage, in the microscopic view, refers to the presence of discontinuities, including micro-cavities and cracks, and its value is also described in a continuum mechanics way, where the size of the RVE is always large enough compared with damage discontinuities [10, 11]. The microcracks initiate when $D=0$ and grow with increasing $D$. When $D=1$, the crack develops to the mesoscale, and occupies the whole surface of the RVE. This is caused by a process of instability which suddenly induces the decohesion of atoms in the remaining resisting area. In a fatigue analysis in practice, damage is assumed to start from 0 and progress to a critical damage value $D_{c}$, instead of 1 , to avoid the effective stress approaching infinity.

The continuum damage mechanics (CDM) approach was widely used to study the fatigue damage under high temperature conditions. Wang et.al. [12] divided the inelastic strain rates for high and low loadings to allow for description of the creep behaviour caused by diffusion and dislocation mechanisms, respectively. The damage process was also separately represented by pure fatigue and pure creep, based on an empirical model. Zhang et.al. [13] investigated the damage evolution under the stress controlled fatigue tests, and proposed a damage model based on the linear damage accumulation theory. The damage parameter is the sum of the creep damage as proposed by Rabotnov [9] and the fatigue damage as proposed by Lemaitre [14]. However, there are several disadvantages. For example, all the partition methods should be used in conjunction with a constitutive model giving different treatments for plasticity and creep. Otherwise, the effects of the plastic deformation and creep may be difficult to differentiate. Moreover, there are strong interactions between creep and fatigue, which increase with the increasing load. The presence of grain boundary cavities caused by creep accelerates the fatigue damage evolution due to embrittlement. On the other hand, the presence of surface cracks caused by fatigue accelerates the creep damage due to the stress concentration effect $[15,16]$.

In this study, a CDM method based on the total strain approach is coupled to the constitutive equations of the cyclic plasticity model to investigate the influence of the damage variable on the Chaboche model behaviour of the P91steel. The constitutive equations of the damage parameter coupled with the Chaboche model are introduced in Section 2. The experimental results, including the fatigue tests and microstructure studies, are shown in Section 3. The damage parameter 
is formulated and analysed in Section 4, while the coding of the ABAQUS UMAT subroutine for the material behaviour is described in Section 5. The results obtained from the finite element analysis are presented and discussed in Section 6. Conclusions and future work are given in Section 7.

\section{Theoretical background}

With the framework of visco-plasticity, the total strain $\boldsymbol{\varepsilon}$ is decomposed into two internal variables, the elastic strain $\boldsymbol{\varepsilon}^{\boldsymbol{e}}$ and the irreversible visco-plastic strain $\boldsymbol{\varepsilon}^{p}$ :

$\varepsilon=\varepsilon^{e}+\varepsilon^{p}$

The elastic strain is calculated using the linear elastic law, as follows:

$\boldsymbol{\varepsilon}^{e}=\frac{1+v}{E} \widetilde{\boldsymbol{\sigma}}-\frac{v}{E} \operatorname{Tr}(\widetilde{\boldsymbol{\sigma}}) \boldsymbol{I}$

where $\widetilde{\boldsymbol{\sigma}}$ is the effective stress defined using the concept of CDM, which is higher than the normal stress measured for a homogeneous continuum, caused by a loss of load bearing area in the RVE, as follows:

$\widetilde{\boldsymbol{\sigma}}=\boldsymbol{\sigma} /(1-D)$

The concepts of kinematic hardening $\boldsymbol{X}$, isotropic hardening $R$ and initial yield stress $\sigma_{y}$ are introduced to describe the yield function $f y$ [17]:

$f y=J\left(\widetilde{\boldsymbol{\sigma}}^{D}-\boldsymbol{X}\right)-R-\sigma_{y}=\left[\frac{3}{2}\left(\widetilde{\boldsymbol{\sigma}}^{D}-\boldsymbol{X}\right):\left(\widetilde{\boldsymbol{\sigma}}^{D}-\boldsymbol{X}\right)\right]^{\frac{1}{2}}-R-\sigma_{y}$

where the function $J$ represents the second invariant of the tensor in the bracket, and $\widetilde{\boldsymbol{\sigma}}^{D}$ is the effective stress deviator. When $f y<0$, the stress response is in the elastic domain; when $f y=0$, the stress response is purely plastic on the yield surface; when $f y>0$, the value of yield function equals the viscous stress $f y=\sigma_{v}$, and the stress response is caused by the visco-plastic behaviour of the material. The macroscopic expressions include a strain hardening term and a dynamic recovery term. The isotropic hardening $R$ represents the size of the elastic domain, and is formulated as follows:

$R=R_{S}[1-\exp (-b p)]+H p$

where $p$ is the accumulated plastic strain, $b$ is the coefficient controlling the changing rate, and $R_{S}$ is the saturated value of isotropic hardening, which represents either isotropic hardening $\left(R_{S}>0\right)$ or isotropic softening $\left(R_{S}<0\right)$. Whether the material hardens or softens depends on the temperature, material composition, and whether the material is pre-hardened. In this paper, cyclic softening occurs, in which dislocations move back and forth to relieve the dislocation accumulation or enable the dislocations to bypass the barrier [18]. The value of saturated isotropic hardening is exponentially related to the plastic strain range, which tends not to vary significantly at higher plastic strain ranges. $H$ is a coefficient used to modify the isotropic hardening in this continuous softening case.

The kinematic hardening variable tensor $X$, related to the internal micro-stress, represents schematically the centre of the elastic domain. The superposition law suggests that there can be several additive kinematic hardening variables $\boldsymbol{X}_{\mathbf{1}}, \boldsymbol{X}_{\mathbf{2}} \ldots$ to consider both long range and short range effects [3]. It was suggested that the slow evolving long range kinematic hardening variable, which results from the dislocation looping mechanism, does not change in the isotropic softening situation $[19,20]$. Therefore, only short range interaction between a dislocation and a single atom or centre of dislocation [21] is considered. Although it is proposed that the short-range kinematic hardening is decomposed into two individual variables, only one kinematic hardening variable is needed under viscoplasticity conditions, since the nonlinearity is already described by the normal viscous effect [22]. The kinematic hardening starts from an initial value $X_{0}$ [19], and the nonlinear evolution law of the kinematic hardening proposed by Armstrong and Frederick [23] is employed for isothermal conditions, such that 
$\dot{\boldsymbol{X}}=\gamma\left(\frac{2}{3} X_{s} \dot{\boldsymbol{\varepsilon}}^{p}-\boldsymbol{X} \dot{p}\right)$

where $X_{S}$ is the saturation value of kinematic hardening and the coefficient $\gamma$ controls the rate of change. For nonisothermal conditions, a temperature rate term is usually used.

The yield stress $\sigma_{y}$ in visco-plasticity formulations is slightly different from the concept in static tests at room temperature, since plastic flow occurs at a much lower stress than the normal yield stress. The flow rule for the plastic strain obeys the normality rule, such that the rate of plastic deformation is the product of the normal direction $\boldsymbol{n}=\frac{3}{2} \frac{\widetilde{\boldsymbol{\sigma}}^{D}-\boldsymbol{X}}{J\left(\widetilde{\boldsymbol{\sigma}}^{D}-\boldsymbol{X}\right)}$ and the accumulated plastic strain rate $\dot{p}$ :

$\dot{\varepsilon}_{i j}^{p}=\boldsymbol{n} \dot{p}$

The value of the accumulated plastic strain rate $\dot{p}$ is defined as

$\dot{p}=\left(\frac{2}{3} \dot{\boldsymbol{\varepsilon}}^{p}: \dot{\boldsymbol{\varepsilon}}^{p}\right)^{\frac{1}{2}}$

In the uniaxial case, the value of the accumulated plastic strain rate equals the absolute value of the strain rate, $\dot{p}=\left|\dot{\varepsilon}^{p}\right|$.

For the visco-plastic material behaviour, it can be calculated from Norton's law such that

$\dot{p}=\left(\sigma_{v} / S_{k}\right)^{n}$

The rate exponent $n$ controls the material sensitivity to plastic deformation, while the drag force $S_{k}$ controls the resistance to plastic deformation. Plasticity is the limiting case when the yield surface $f y=0$, and the value of the accumulated plastic strain rate $\dot{p}$ can be obtained by solving the equation $f y=0$.

In low cycle fatigue, the number of cycles leading to macrocrack nucleation usually dominates the total life [24]. This is termed as the total life approach, in which the number of cycles to failure is assumed to be the number of cycles to crack initiation. The number of cycles to failure $N_{f}$ can be derived from the Coffin-Manson relationship in the uniaxial case, such that

$N_{f}=\left(\Delta \varepsilon^{p} / 2 c_{F}\right)^{\gamma_{F}}$

in which $\Delta \varepsilon^{p}$ is the steady state plastic strain range, $\gamma_{F}$ and $c_{F}$ are both material constants. In the multiaxial case, the accumulated plastic increment $\Delta p$ is used instead of $\Delta \varepsilon^{p}$. A damage evolution curve is normally considered as a function of the life portion $N / N_{f}$, denoted as $L$, so that the damage evolution depends on the loading sequence and assumes nonlinear damage accumulation. Damage initiation criteria exist in the literature $[10,14]$, such as the accumulated plastic deformation or accumulated hysteresis energy criteria. However, the values at those damage initiation criteria are also strain range dependent for $\mathrm{P} 91$ at a temperature of $600^{\circ} \mathrm{C}$, as shown in later experimental results in Section 3.1. In this study, it is assumed that damage initiates at the very beginning of the fatigue tests. A phenomenological description of the damage parameter against the life portion $L$ is used based on the experimental observation that damage evolution for all the strain ranges was consistent against the life portion, as follows:

$D=\sinh \left(C_{2} L\right) / C_{1}$

where $C_{1}$ and $C_{2}$ are material constants. The life portion starts when $L=0$ and ends when $L=1$, and its incremental rule is defined as:

$d L / d N=1 / N_{f}\left(\Delta \varepsilon^{p}\right)$

\section{Experimental work}

The material used in this work was a 9\% Cr steel alloy (P91), and its composition is shown in Table 1.

Table 1. Chemical composition for P91 steel (wt\%) [7] 


\begin{tabular}{|c|c|c|c|c|c|c|c|c|c|c|}
\hline $\mathrm{Cr}$ & $\mathrm{Mo}$ & $\mathrm{C}$ & $\mathrm{Si}$ & $\mathrm{S}$ & $\mathrm{P}$ & $\mathrm{Al}$ & $\mathrm{V}$ & $\mathrm{Nb}$ & $\mathrm{N}$ & $\mathrm{W}$ \\
\hline 8.60 & 1.02 & 0.12 & 0.34 & $<0.002$ & 0.017 & 0.007 & 0.24 & 0.070 & 0.060 & 0.03 \\
\hline
\end{tabular}

Several strain-controlled isothermal fatigue tests were conducted at a temperature of $600{ }^{\circ} \mathrm{C}$ to investigate the stress-strain relationship and the damage effect in the macro-scale by Saad[7]. The tests were performed at a strain rate of $0.1 \% / \mathrm{s}$ and with strain ranges of $\pm 0.5 \%, \pm 0.4 \%, \pm 0.25 \%$ and $\pm 0.2 \%$. The test specimen geometry follows the British Standard ISO 12111:2011(E) [25]. Another set of experiments with the same testing temperature at $600{ }^{0} \mathrm{C}$ with a dwell holding time of 120 s at the maximum strain were conducted.

In addition, the fatigue test with the strain range of $\pm 0.5 \%$ was interrupted at cycle numbers 200 and 400 to allow for microstructure investigations for the as-received specimen, the interrupted specimens, and the specimen at failure. Samples were extracted from the longitudinal direction of the specimen's gauge section for the Philips/FEI XL-30 scanning electron microscope (SEM) to investigate damage in the mesoscale. Meanwhile, in order to understand the mechanism responsible for the cyclic stress response, the JOEL 2000FX Transmission election microscopy (TEM) at $200 \mathrm{kV}$ is also used for the samples taken perpendicularly to the loading axis. TEM is capable of studying the microstructure changes at a higher magnification, to observe features such as lath structure and dislocations.

\subsection{Fatigue test}

The direct outputs from the fatigue test include the stress-strain curves for each cycle, as well as the maximum and minimum stress evolution against cycle number. The low cycle fatigue life curves were plotted as stress amplitude $(\Delta \sigma / 2)$ of each cycle against cycle number in Figure 1(a) which indicates that the stress amplitude keeps decreasing, except for the first few cycles. Moreover, as the strain ranges decrease, the cyclic maximum stress response decreases and the fatigue life becomes longer for the strain range of $\pm 0.5 \%, \pm 0.4 \%$ and $\pm 0.25 \%$. An exception is the fatigue test with a strain range of $\pm 0.2 \%$, for which the number of cycles to failure is greater than 10000 , and therefore the fatigue behaviour is assumed to be elasticity dominant. This is an indication that the strain range of $\pm 0.2 \%$ may represent a critical value between low and high cycle fatigue.

In the British Standard used in this test [25], the failure criteria defined for the continuously softening materials is when there is a specified force drop from the projected straight line locus of peak tensile stress versus cycles. In this study, a $10 \%$ force drop is selected as the failure criterion. Therefore, a critical damage value of $D_{c}=0.1$ is chosen in the FE analysis. This is the reason why the stress amplitude decreases at a steady rate, and the stress amplitude could be related to the cycle number $N$ in a linear pattern, as shown in Figure 2(a), as follows:

$\Delta \sigma / 2=C_{3} N+C_{4}$

where $C_{3}$ and $C_{4}$ are material constants. The material is assumed to fail when the experimental stress amplitude curve meets the straight line described by the following equation:

$\Delta \sigma / 2=C_{3} N+C_{4} \times 90 \%$

The obtained number of cycles to failure $N_{f}$ were $608,1467,3614$ and 10659 for the strain ranges of $\pm 0.5 \%, \pm 0.4 \%$, $\pm 0.25 \%$ and $\pm 0.2 \%$ respectively. They were related to the plastic strain range by the Manson-Coffin relationship as in Equ. (10) in the uniaxial case, and the material constants obtained were $c_{F}=29.403$ and $\gamma_{F}=0.57$, as shown in Figure 2(b).

From the evolution of the stress amplitude for all these strain ranges, it could be observed that there is a fast initial decrease of stress amplitude before $0.2 N_{f}$, followed by a steady linear decrease during cycle numbers between 
approximately $0.2 N_{f}$ and $0.8 N_{f}$. Finally, there is a sudden decrease of stress amplitude after $0.8 N_{f}$. The evolution of the mean stress is rather small (less than $5 \mathrm{MPa}$ and not shown here), which is negligible compared with the stress amplitude.

The detailed stress-strain loops for cycle numbers 1, 101, 201, 301, 401, 501 and 601, 655 (final cycle number) for the strain range of $\pm 0.5 \%$ are plotted in Figure $1(\mathrm{~b})$, and the cycle number could be identified easily by the decreasing maximum stress. These stress-strain curves are smooth without a sudden change at yielding, which is caused by the viscous effect. There is a clear change of slope in the elastic region when the number of cycles is 601 or $655\left(N_{f}\right)$, but not much of a clear change before the cycle number $501\left(0.8 N_{f}\right)$. Since damage could also be regarded as the degradation of Young's modulus, it is reasonable to postulate that the final decrease of stress amplitude was caused by the acceleration of damage propagation, which could be observed macroscopically after 501 cycles.

The plastic strain increment of each cycle can be obtained as twice of the distance between the interception points of the stress-strain loops and the x-axis. They are plotted against the life portion in Figure 3(a), which shows that the variation of the plastic strain range was small, especially after the initial softening period. The accumulated plastic deformation was one of the quantities regarded as the damage initiation indicator in the Chaboche viscoplasticity model. They are plotted in Figure 3(b), which shows that for the same life portion, the accumulated plastic deformations are not the same, which may depend on the number of cycles to failure. Similar results also apply to the accumulated hystersis energy. Therefore, both of these two damage initiation indicators may not be suitable in this study.
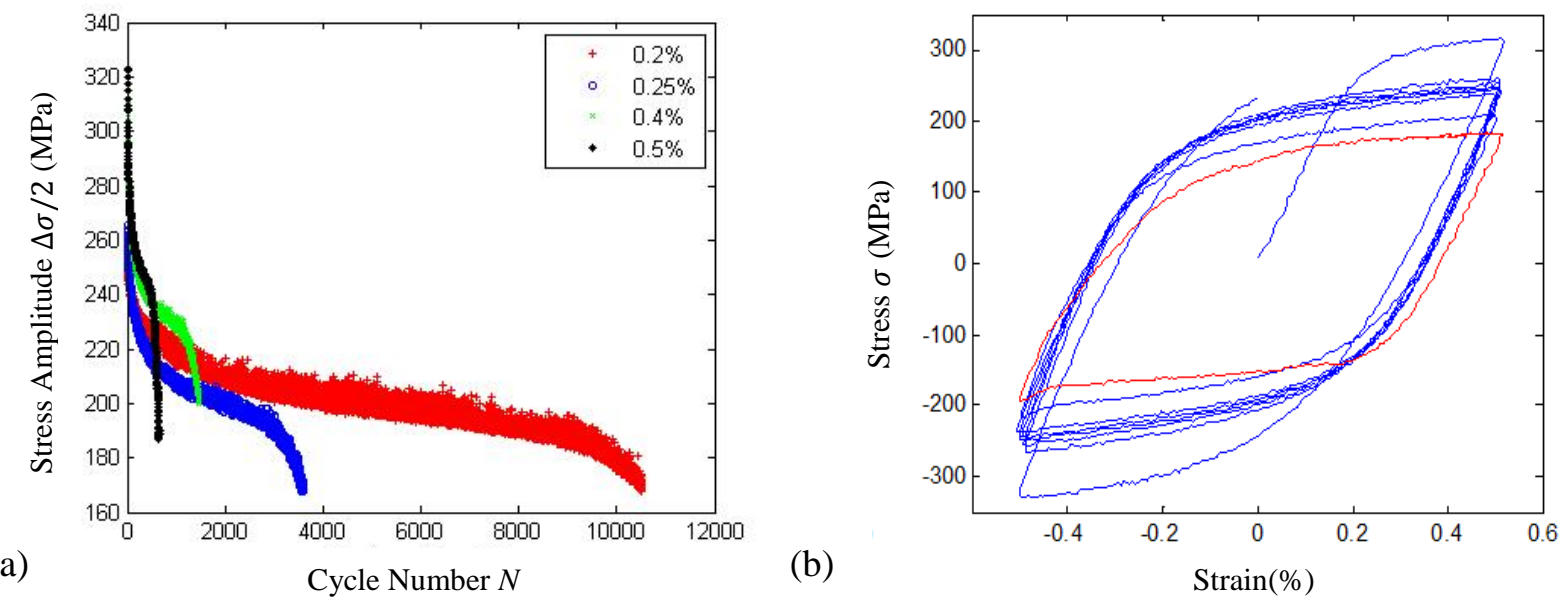

Figure.1. (a) Stress amplitude $\Delta \sigma / 2$ against cycle number for strain ranges of $\pm 0.5 \%, \pm 0.4 \%, \pm 0.25 \%$ and $\pm 0.2 \%$. (b) Stress-strain curves for strain range at $\pm 0.5 \%$ at the cycle number 1, 101, 201, 301,401, 501 and 601,655 (the final loop) [7] 

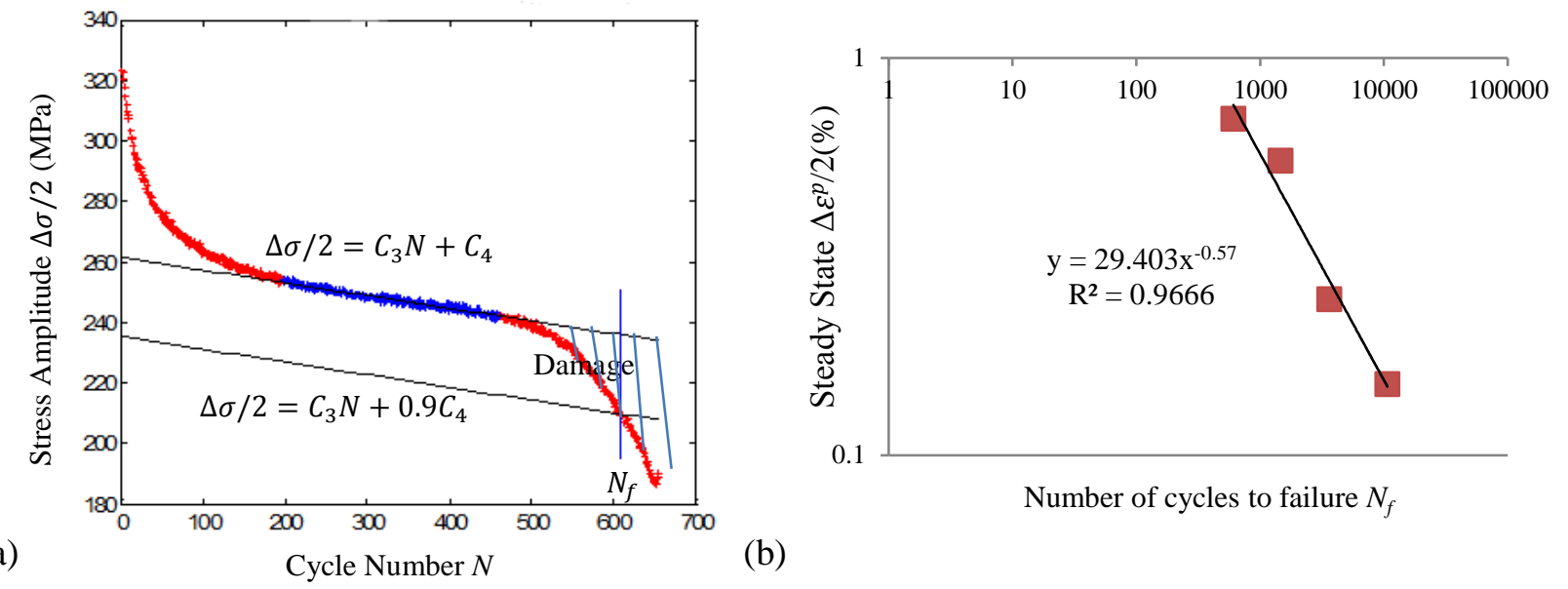

Figure 2. (a) Determination of number of cycle to failure $N_{f}$ and damage quantity; (b) Determination of material constants in the Manson-Coffin relationship at $600{ }^{\circ} \mathrm{C}$.

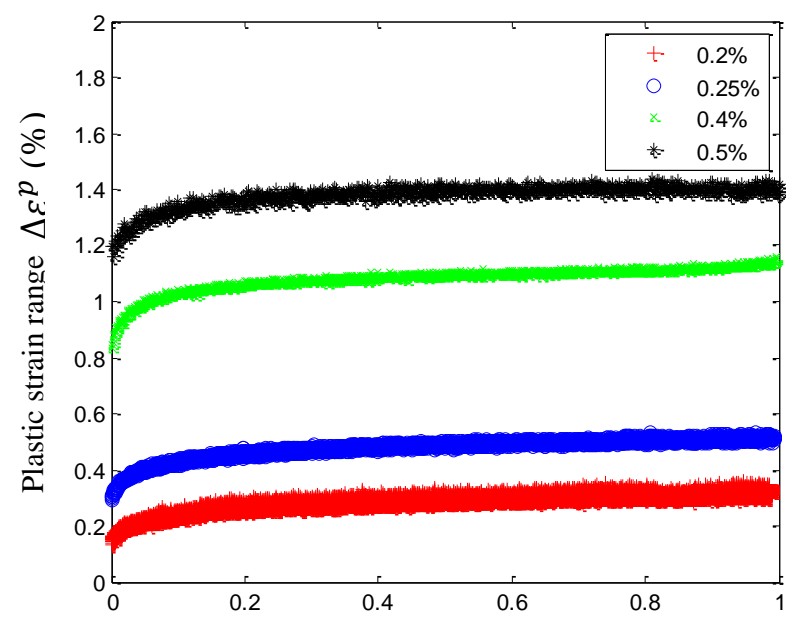

(a) Life portion $N / N_{f}$

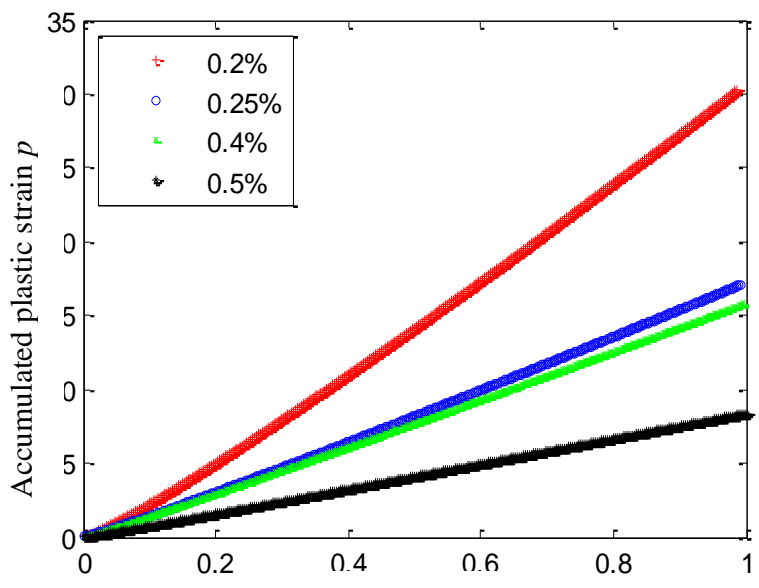

Life portion $N / N_{f}$

Figure 3. (a) Plastic strann range $\Delta \varepsilon^{r}$ agannst the life portion $N / N_{f}$; (b) Accumulated plastic strain $p$ evolution against the life portion $N / N_{f}$

\subsection{Microstructure study}

The microstructure tests, including SEM and TEM, were conducted for the as-received specimen, the interrupted specimens at cycle numbers $200\left(0.33 N_{f}\right)$ and $400\left(0.66 N_{f}\right)$, and the specimen at failure, for the strain range of $\pm 0.5 \%$.

It is observed from the SEM results [7] that there is no obvious microstructure change before the $200^{\text {th }}$ cycles (Figure 4). A crack initiates before the $400^{\text {th }}$ cycle at the specimen's surface at two locations, and many microcracks with a length of about a grain size were observed at the $400^{\text {th }}$ cycle (Figure $5 \mathrm{a}$ ). When the force drops by over $10 \%$ at failure, the crack lengths are about 10 grain sizes (Figure 5b). Accurate recording of damage initiation would require additional acoustic systems.

Bright field TEM results are shown in Figures 6 and 7 [7] to examine the microstructural mechanics responsible for the cyclic softening mechanics for P91 at high temperature. Some features could be observed under bright field TEM, which are regarded as obstacles to overcome in producing slip, and contribute to material softening. The first is the subgrain microstructure, named as martenstic lath. They cannot be observed under SEM since the misorientations between subgrain boundaries are small $\left(<5^{\circ}\right)$, and these low angle boundaries could also be regarded as arrays of edge and screw type 
dislocations. In addition, dislocations inside the subgrains could be observed as short straight lines pinned at subgrain boundaries. A small fraction of dislocations inside and at the subgrain boundaries is regarded as mobile dislocations contributing to plastic deformation, whereas the majority of dislocations hinder plastic deformation [26]. The dark sites in the TEM picture are normally precipitates at the subgrain boundaries or caused by some pollution during the experiment. Better results of the precipitate should be achieved by the dark field TEM.

Both subgrain size and dislocation density could be roughly calculated by the line intercept method [27]. For example, the average dislocation density $\rho$ inside the subgrains is calculated by drawing five random lines through the TEM images with total length $L_{r}$ and counting the number of the intersection points $N$, with the following equation:

$\rho=N / L_{r} t$

in which $t$ is the thickness of the TEM foil. The evolution of subgrain size and dislocation density obtained from TEM results are plotted in Figure 8a.

The as-received sample exhibits small and inconsistent subgrain sizes, and high dislocation density. As cycles increase to cycle number 200 when the stress amplitude softens initially, the lath structure becomes longer, and the subgrain size increases by around 33\%. There are thus less low angle boundaries, and a lower dislocation density. Dislocation annihilation occurs since dislocations located inside the subgrain tend to move towards the low angle boundaries of the opposite sign until the distance is less than a critical dislocation annihilation value. Slower evolutions of the subgrain size and dislocations density were observed after cycle number 200 to failure.

(a)

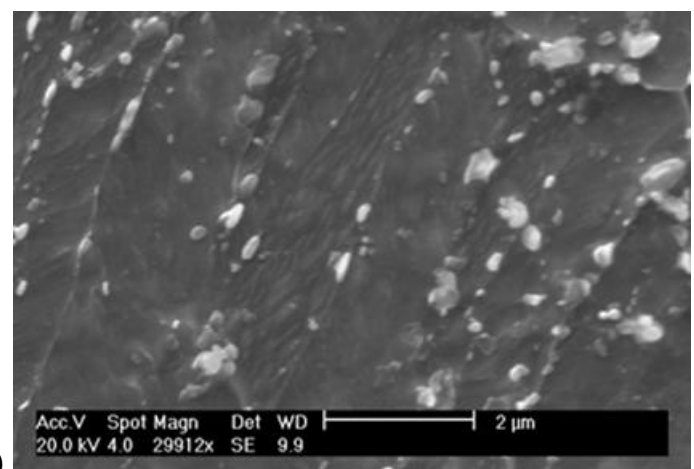

(b)

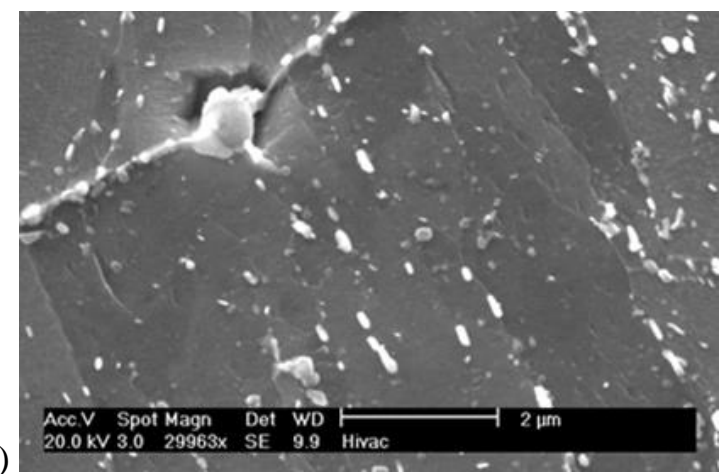

Figure 4. SEM results at the surface of the P91 specimen in a fatigue test at strain range of $\pm 0.5 \%$ : (a) the as-received material and (b) at the $200^{\text {th }}$ cycles $\left(0.33 N_{f}\right)$

(a)

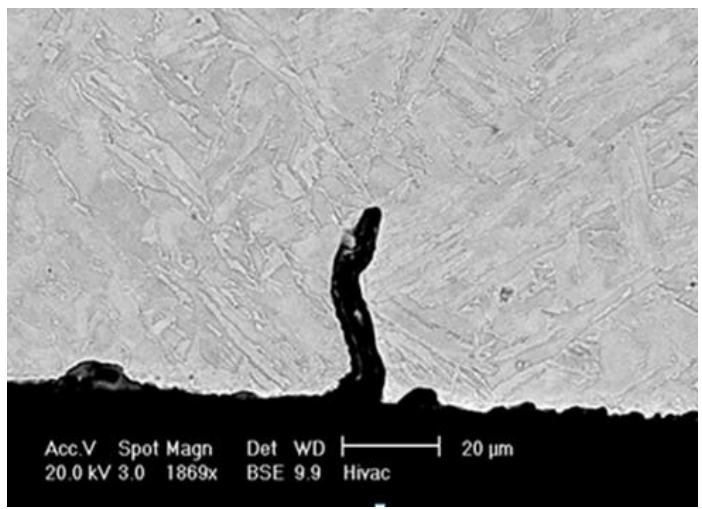

(b)

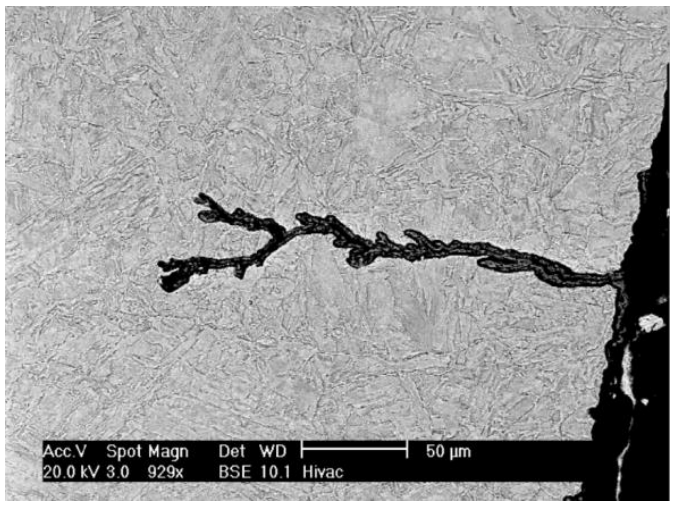

Figure 5. SEM results at the surface of the P91 specimen in a fatigue test at strain range of $\pm 0.5 \%$ : (a) Microcracks observed at the $400^{\text {th }}$ cycles $\left(0.66 N_{f}\right)$; (b) Mesocracks observed at failure 

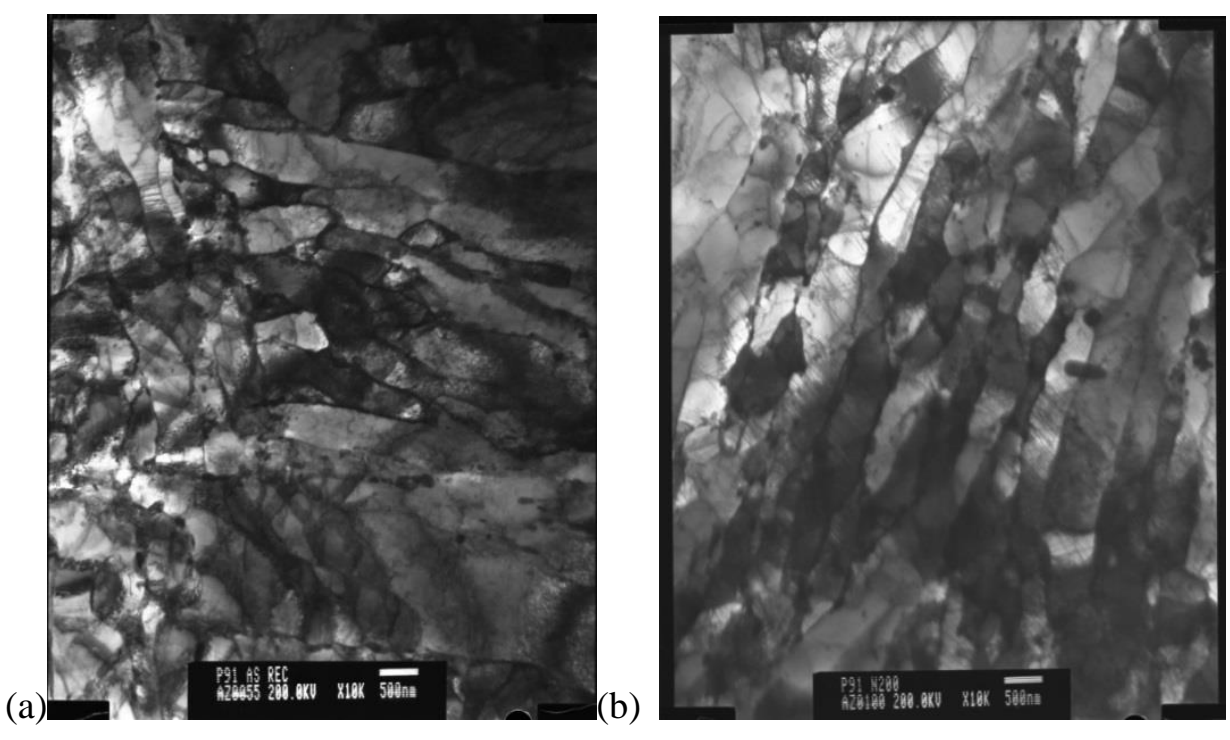

Figure 6. Bright field TEM results of the P91 specimen in a fatigue test at strain range of $\pm 0.5 \%$ (a) the as-received material and (b) at the $200^{\text {th }}$ cycles $\left(0.33 N_{f}\right)$
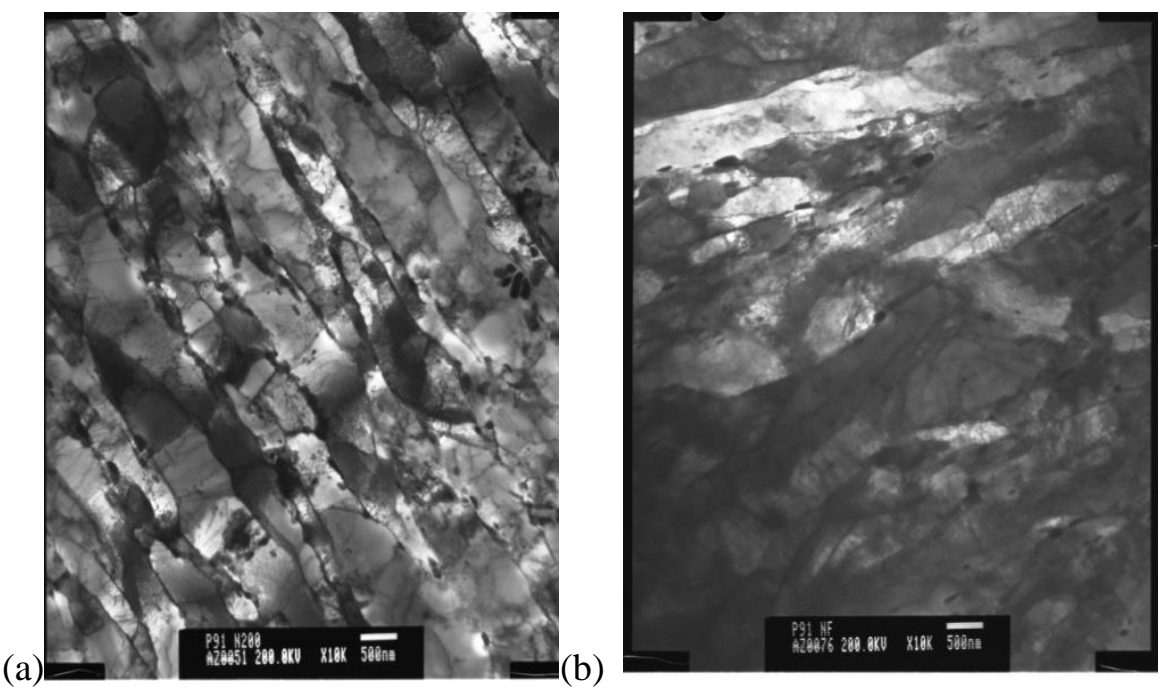

Figure 7. Bright field TEM results of the P91 specimen in a fatigue test at strain range of $\pm 0.5 \%$ : (a) at the $400^{\text {th }}$ cycles $\left(0.66 N_{f}\right)$; (b) at failure

\section{Damage formulation}

In the macroscopic scale, damage could be viewed as the degradation of Young's modulus due to the loss of load bearing area. Therefore, a typical method to obtain damage evolution is to determine the effective Young's modulus $\tilde{E}$ by the following equation:

$\tilde{E}=\Delta \sigma /\left(\Delta \varepsilon-\Delta \varepsilon_{p}\right)$

The damage parameter can be determined via the following equation:

$\mathrm{D}=1-\tilde{E} / E$

The result is shown in Figure 8(b). There is initial stiffening during the first tens of cycles, which is assumed to be caused by thermal fluctuation during the experiment start-up. Alternatively, since in a strain-controlled test, it could be assumed that after the initial softening period, the plastic strain range is constant and the value of damage before $0.6 N_{f}$ is regarded 
as zero, damage evolution could be obtained by measuring the stress amplitude percentage degradation from the linear regression curve of the stress amplitude in the linear softening stage, by the following equation:

$$
D=1-\Delta \sigma / 2\left(C_{3} N+C_{4}\right)
$$

The damage evolution for the strain range of $\pm 0.5 \%$ obtained from the above two methods follows nearly the same trend. In addition, the damage evolutions for the strain ranges of $\pm 0.5 \%, \pm 0.4 \%, \pm 0.25 \%$ and $\pm 0.2 \%$ are also consistent against the life portion. As mentioned before, the critical damage $D_{c}$ is defined to be 0.1 here. The evolution curves are fitted to the $\sinh$ function of the life portion, as in Equ.(11), which gives $C_{1}=187667$ and $C_{2}=10.6$. The damage and life portion evolution laws are written as follows:

$D=\sinh (10.6 L) / 187667$ and $d L / d N=1 / N_{f}\left(\Delta \varepsilon^{p}\right)=\left(58.806 / \Delta \varepsilon^{p}\right)^{0.57}$

(a)
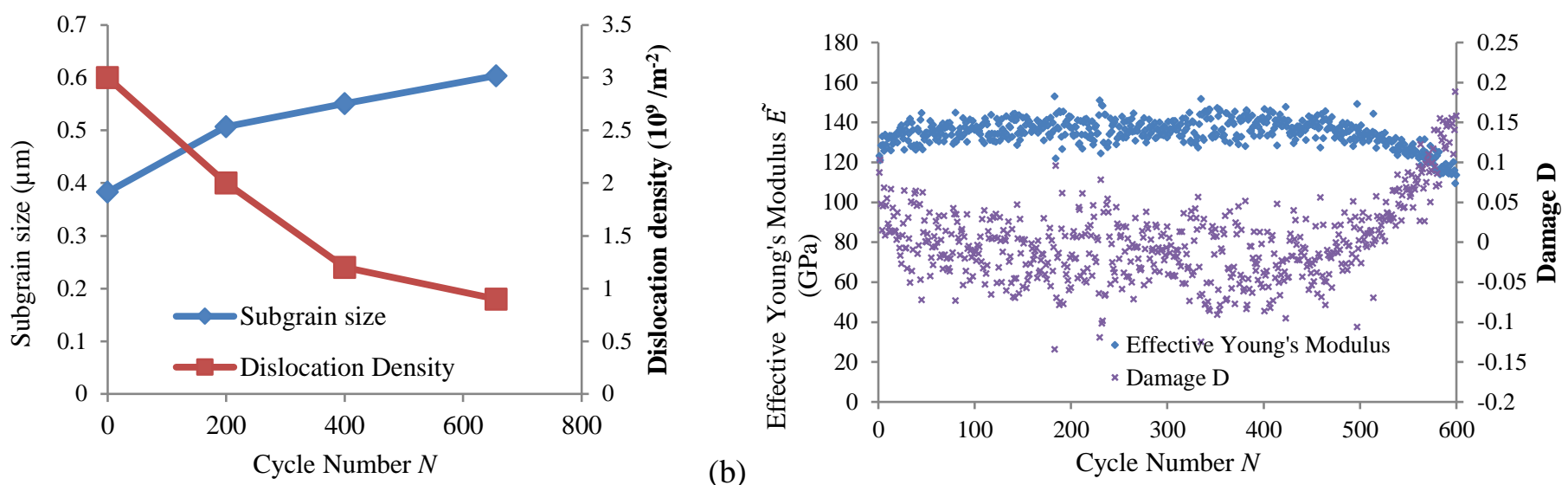

Figure 8. (a) Subgrain size and dislocation density evolution based on the TEM results for the strain range of $\pm 0.5 \%$;(b) Evolution of the effective Young's modulus $\tilde{E}$ and damage $D$ against cycle number $N$

\section{Finite Element Implementation}

\subsection{UMAT subroutine}

The commercially available software ABAQUS is used to conduct the FE analysis. The built-in FE solver of ABAQUS Standard is able to process the inputs of geometry and loading conditions, and to calculate the stresses and strains using an implicit scheme. For material behaviour models not available in ABAQUS, for example, the Chaboche visco-plasticity model, a UMAT subroutine can be used by the users to code the material behaviour. In order for the UMAT to work inside ABAQUS Standard, which uses the Newton-Raphson method to solve nonlinear problems, the user needs to provide updated stresses and the Jacobian matrix (change of stress increment with respect to strain increment) based on the previous material condition at every strain increment. Several parameters, including damage, accumulated plastic strain, are defined as solution-dependent state variables (SDV in ABAQUS), which could be directly viewed in the output file of ABAQUS.

The UMAT subroutine is suitable for use in axisymmetric, 2D plane strain and 3D problems. In the Chaboche viscoplasticity model, plastic strain, kinematic hardening and isotropic hardening are fully coupled in the constitutive equations. An elastic predictor-plastic corrector method is used to update the stress in the coding [28].

The first step is to assume that there is no plastic deformation $(\Delta p=0)$, and the stress increment is caused by elastic deformation only from time point $n$ to $n+1$, and thus there is no change in isotropic and kinematic hardening. It is assumed that the trial flow direction of the elastic-predictor is the same as the flow direction, as follows:

$\widetilde{\boldsymbol{\sigma}}^{p r}=\widetilde{\boldsymbol{\sigma}}_{n}+\tilde{\boldsymbol{J}}^{e} d \boldsymbol{\varepsilon}$ 
where $\tilde{\boldsymbol{J}}^{\boldsymbol{e}}$ is the effective elastic Jacobian matrix, the passed-in variable $\widetilde{\boldsymbol{\sigma}}_{n}$ is the effective stress incorporating the damage parameter for the last increment, and $\widetilde{\boldsymbol{\sigma}}^{p r}$ is the stress at time increment $n+1$ obtained by the elastic-predictor. Since the material behaviour is assumed to be fully elastic as an initial estimate, there is no change in isotropic and kinematic hardening variables. However, if the material behaviour in the specific increment $n$ is unable to accommodate the excessive stress, the elastic predictor brings the yield function $f y$ out of the yield surface, such that

$f y=J\left(\widetilde{\boldsymbol{\sigma}}^{p r}-\boldsymbol{X}_{n}\right)-R_{n}-\sigma_{y}>0$

The second step is to use the plastic corrector to correct the accumulated plastic deformation, and therefore the stress, to make the yield function satisfy the Norton's law $\phi(\Delta \mathrm{p}, \boldsymbol{X}, R)$ when there is creep. Since the elastic-predictor normally under-estimates the plastic deformation and over-estimates the stress response at time point $n+1$, the plastic-corrector is in the reversed direction of the flow direction. The Newton-Raphson iteration method is used to solve for the incremental accumulated plastic deformation $\Delta p$ by minimising the objective residual function $f_{\text {res }}$

$f_{\text {res }}=\phi(\Delta \mathrm{p}, \boldsymbol{X}, R)-\Delta p / \Delta t$

where

$\phi(\Delta \mathrm{p}, \boldsymbol{X}, R)=\dot{p}_{n+1}=\left[\left(J\left(\widetilde{\boldsymbol{\sigma}}^{p r}-\boldsymbol{X}_{n}\right)-R_{n}-\sigma_{y}\right) / S_{k}\right]^{n}$

Expanding Equation (22) by Taylor's expansion,

$f_{\text {res }}+\frac{\partial f_{\text {res }}}{\partial \Delta \mathrm{p}} \delta \Delta \mathrm{p}+\frac{\partial f_{\text {res }}}{\partial \mathrm{R}} \delta R+\frac{\partial f_{\text {res }}}{\partial \boldsymbol{X}} \delta \boldsymbol{X}=0$

The partial differential can be unified as

$\frac{\partial f_{r e s}}{\partial \mathrm{R}}=\frac{\partial \phi}{\partial R}=-\frac{\partial \phi}{\partial \sigma_{e}}$

$\frac{\partial f_{r e s}}{\partial \boldsymbol{X}}=\frac{\partial \phi}{\partial \boldsymbol{X}}=-\frac{\partial \phi}{\partial \sigma_{e}} \frac{\partial \sigma_{e}}{\partial \boldsymbol{X}}=-\frac{\partial \phi}{\partial \sigma_{e}} \frac{3}{2} \frac{\widetilde{\boldsymbol{\sigma}}^{p r}-\boldsymbol{X}_{n}}{J\left(\widetilde{\boldsymbol{\sigma}}^{p r}-\boldsymbol{X}_{n}\right)}=-\frac{\partial \phi}{\partial \sigma_{e}} \boldsymbol{n}$

$\frac{\partial f_{r e s}}{\partial \Delta \mathrm{p}}=\frac{\partial \phi}{\partial \Delta \mathrm{p}}-\frac{1}{\Delta t}=-3 G \frac{\partial \phi}{\partial \sigma_{e}}-\frac{1}{\Delta t}$

where $\sigma_{e}$ is the equivalent stress expressed as

$\sigma_{e}=J\left(\boldsymbol{\sigma}_{n+1}^{D}-\boldsymbol{X}_{n+1}\right)=J\left(\widetilde{\boldsymbol{\sigma}}^{p r}-\boldsymbol{X}_{n+1}\right)-(3 G) \Delta \mathrm{p}$

Rearranging Equ.(24), gives

$\delta \Delta \mathrm{p}=\frac{f_{\text {res }}}{\frac{1}{\Delta t}-\frac{\partial \phi}{\partial \Delta \mathrm{p}}-\frac{\partial \phi \partial R}{\partial R \partial \Delta \mathrm{p}}-\frac{\partial \phi \partial X}{\partial X \partial \Delta \mathrm{p}}}=\frac{f_{r e s}}{\frac{1}{\Delta t}+\left(3 G+\frac{\partial R}{\partial \Delta \mathrm{p}}+n: \frac{\partial X}{\partial \Delta \mathrm{p}}\right) \frac{\partial \phi}{\partial \sigma_{e}}}$

and

$R+\Delta R=Q\left(1-\mathrm{e}^{-\mathrm{b}(\mathrm{p}+\Delta p)}\right)+H(p+\Delta p) \stackrel{\text { yields }}{\longrightarrow} \frac{\partial R}{\partial \Delta \mathrm{p}}=b\left(R_{s}-R\right)+H(1+b p)$

$\frac{\partial \boldsymbol{X}}{\partial \Delta \mathrm{p}}=\frac{2}{3} \gamma X_{s} \boldsymbol{n}-\gamma \boldsymbol{X} \stackrel{\text { yields }}{\longrightarrow} \boldsymbol{n}: \frac{\partial \boldsymbol{X}}{\partial \Delta \mathrm{p}}=\frac{2}{3} \gamma X_{s} \boldsymbol{n}: \boldsymbol{n}-\gamma \boldsymbol{X}: \boldsymbol{n}=\gamma X_{S}-\gamma \boldsymbol{X}: \boldsymbol{n}$

The accumulated plastic deformation $\Delta p$ is updated by $\Delta p+\delta \Delta \mathrm{p}$. The final step is to update the isotropic hardening, kinematic hardening, life portion, damage parameter and stress, as follows:

$R_{n+1}=R_{n}+b\left(R_{S}-R_{n+1}\right) \Delta p+H(1+b p) \Delta p$

$\boldsymbol{X}_{n+1}=\left(\boldsymbol{X}_{n}+\frac{2}{3} \gamma X_{S} n \Delta p\right) /(1+\gamma \Delta p)$ 
$L_{n+1}=L_{n}+N / N_{f}\left(\Delta \varepsilon^{p}\right)$

$D_{n+1}=\sinh \left(C_{2} L_{n+1}\right) / C_{1}$

$\boldsymbol{\sigma}_{n+1}=\tilde{\boldsymbol{J}}^{e}\left(\Delta \boldsymbol{\varepsilon}-\Delta \boldsymbol{\varepsilon}^{p}\right)$

The stress and the Jacobian matrix are updated at the start of each time interval based on the pass-in information and the material constitutive equations. The ABAQUS implicit solver aims to minimise the difference between the external force and the internal force at a convergence rate determined from the Jacobian matrix. The Jacobian matrix to be updated in the UMAT subroutine is the same as the elastic Jacobian matrix $\tilde{\boldsymbol{J}}^{e}$ to simplify the coding, since it only accounts for the convergence rate of the ABAQUS FE solver. The flowchart of the implementation of the UMAT subroutine for the viscoplasticity model is shown in Appendix A.

\subsection{Determination of the material properties}

There are several material constants in the above set of equations, which are defined in the UMAT subroutine. The stress partition method was coded to give an initial estimate of the material constants, and further optimisation procedures are needed for better accuracy, due to the scattering of the stress partition results.

\subsubsection{Stress partition method}

For the isothermal fatigue test under high temperature, the stress response is located at the outside of the yield circle, and its value in the uniaxial case is the sum of the stress components, $X, R, \sigma_{y}$ and $\sigma_{v}$. The unloading curve can be partitioned to these stress components based on equations (37)-(39), as shown in Figure 9(a). Upon unloading, the yield function is still greater than zero, there would be a fast initial decrease of the stress response, and the stress-strain slope may be even larger than the effective Young's modulus $\tilde{E}[29,30]$. The method used here to differentiate between the elastic domain and viscous part is based on using the coefficient of determination $R^{2}$, from which the maximum stress for the elastic domain $\sigma_{e}^{\max }$ could be obtained. The method to differentiate bewteen the elastic domain and the reversed visco-plastic deformation is based on the offset strain $\varepsilon_{\text {offset }}$, from which the minimum stress $\sigma_{e}^{m i n}$ for the elastic domain could be obtained. The size and centre of the elastic domain and the viscous stress values, could be obtained based on the Cottrell's method [21].

$X=\left(\sigma_{e}^{\max }+\sigma_{e}^{\min }\right) / 2$

$\sigma_{v}=\sigma^{\max }-\sigma_{e}^{\max }$

$R+\sigma_{y}=\left(\sigma_{e}^{\max }-\sigma_{e}^{\min }\right) / 2$

These stress partition procedures were coded in a MATLAB script and were applied to the stress-strain curve for the strain range of $\pm 0.5 \%$ under a temperature of $600{ }^{\circ} \mathrm{C}$. Actually, the final results would be influenced by the extent of the control criterion significantly. For example, the elastic domain size increases when $\varepsilon_{\text {offset }}$ increases. In this study, the control criterion is set as $R^{2}=0.99$ and $\varepsilon_{\text {offset }}=0.1 \%$.

The radius and centre of the elastic domain are plotted against the number of cycles $N$ in Figure 9(b), as well as the viscous stress $\sigma_{v}$. The obtained stress components are quite scattered; however, the general trend can be easily observed. The initial softening of the stress amplitude is attributed to the initial fast decrease of both isotropic and kinematic hardening. The linear decrease of the stress amplitude is caused by the steady decrease of isotropic hardening, and kinematic hardening is already saturated with regard to the accumulated plastic deformation. The final decrease of stress amplitude is caused by Young's modulus degradation, which also results in the decrease of the size of the elastic domain. Throughout the fatigue life, not much change was observed for the viscous stress. 
All these stress components are curved fitted to equations (5) and (6) to give the initial estimate of the material constants for the Chaboche visco-plasticity model in Table 2. However, further optimisation procedures are still needed to accurately fit the experimental data.

(a)

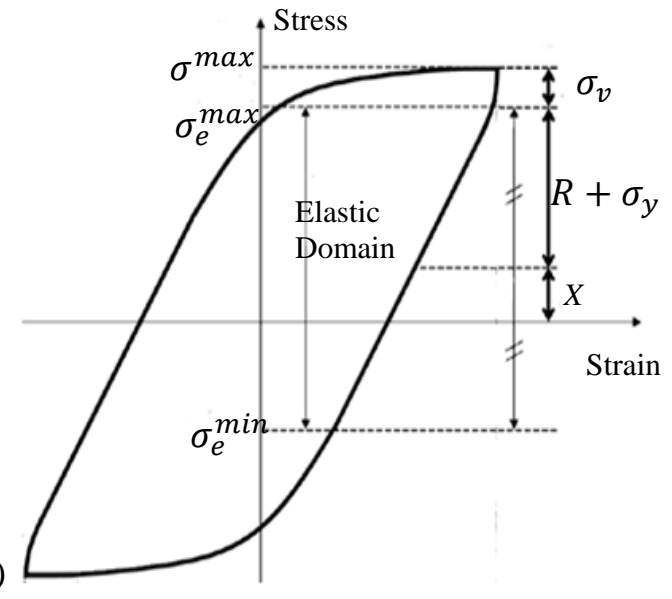

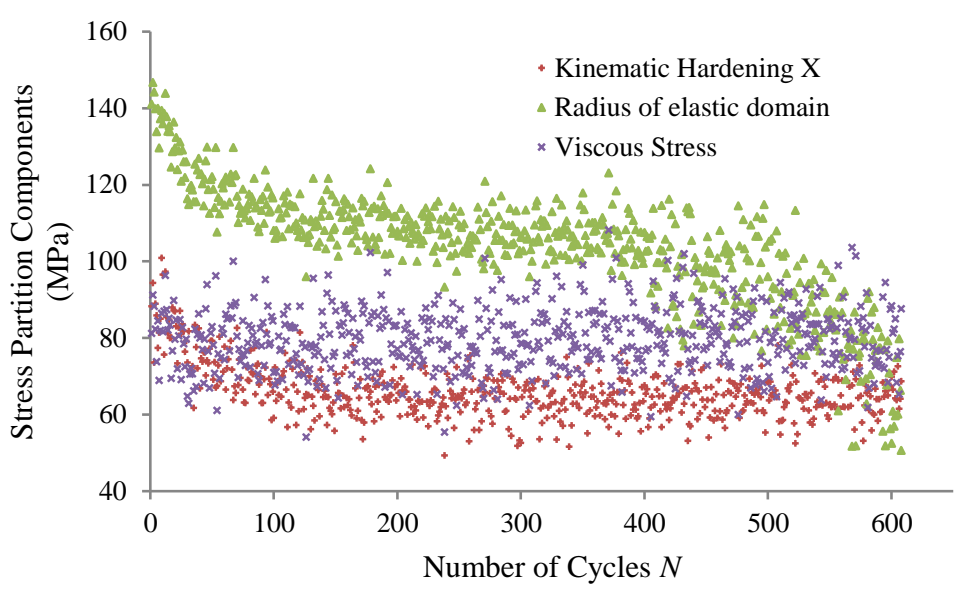

Figure 9. (a) Stress partition method [31]; (b) Evolution of the stress components by the stress partition method, including radius of elastic domain $R+\sigma_{y}$, kinematic hardening $X$ and viscous stress $\sigma_{v}$

\subsubsection{Optimisation procedure}

In order to accurately predict the value of the drag force and the exponent in the Norton's law, another set of experiments with the same testing temperature at $600{ }^{\circ} \mathrm{C}$ with a dwell holding time of $120 \mathrm{~s}$ at the maximum tensile strain were conducted. The optimisation process is available in the toolbox of MATLAB by the function Isqcurvefit, to find an array of unknown material constants $\boldsymbol{q}$ that fit a series of experimental data ( $x$ data,ydata). It is conducted by minimising the difference between the FE simulation results $F(q, x$ data $)$ and several objective functions ydata, based on a nonlinear least-squares algorithm. The objective functions include both the experimental fatigue data and stress relaxation data obtained in the tests with dwell time [7]. The flowchart of the curve fitting process is shown in Appendix B.

As mentioned earlier, isotropic hardening is dependent on the plastic strain range. However, since the initial softening period is relatively short, and nearly no plastic strain range change is observed after the initial softening stage (Figure 2(b)), the material constants for isotropic hardening are considered as constant for each fatigue test in the optimisation procedure.

During the optimisation procedure for the fatigue results at the strain range of $\pm 0.5 \%$, all of the material constants, excluding the elastic material constants are regarded as variables. The objective function is the detailed stress evolution for the first 50 cycles, and the maximum and minimum stress evolution for the next 350 cycles, during the stress amplitude linear softening stage. The initial estimate is based on the stress partition method listed in Table 2.

Table 2. Material constants for $\mathrm{P} 91$ at temperature of $600{ }^{\circ} \mathrm{C}$ and for the strain range of $\pm 0.5 \%$

\begin{tabular}{|c|c|c|c|c|c|c|c|c|c|c|c|}
\hline & $\begin{array}{c}E \\
(\mathrm{MPa})\end{array}$ & $v$ & $\begin{array}{c}\sigma_{y} \\
(\mathrm{MPa})\end{array}$ & $\begin{array}{c}K_{S} \\
\left(\mathrm{MPa} \mathrm{s}^{1 / \mathrm{n}}\right)\end{array}$ & $n$ & $b$ & $\begin{array}{c}R_{S} \\
(\mathrm{MPa})\end{array}$ & $\begin{array}{c}X_{0} \\
(\mathrm{MPa})\end{array}$ & $\begin{array}{c}X_{S} \\
(\mathrm{MPa})\end{array}$ & $\gamma$ & $H$ \\
\hline $\begin{array}{c}\text { Initial } \\
\text { Estimate }\end{array}$ & 125000 & 0.3 & 141 & 1000 & 2 & 2.07 & -26.8 & 31.6 & -23.2 & 1.29 & -1.7 \\
\hline $\begin{array}{c}\text { Optimised } \\
\text { result }\end{array}$ & 125000 & 0.3 & 120 & 6339 & 2.02 & 10 & -66 & 0.24 & -10 & 1.29 & -1.73 \\
\hline
\end{tabular}

For the optimisation procedure for fatigue results at the strain ranges of $\pm 0.4 \%, \pm 0.25 \%$ and $\pm 0.2 \%$, the cyclic behaviour for each cycle is not regarded as a priority, since the aim of the study concentrates on the peak stress response 
in the full fatigue life. The objective functions are the maximum and minimum stresses for the fatigue life before the end of the linear softening stage. Since the linear softening period is much longer than the initial fast softening period, the optimisation procedure places more weighing factor to the results of the linear softening period automatically. The initial estimates are the same as the optimised results of the material constants for the strain range of $\pm 0.5 \%$, and the variables are for isotropic hardening only. The optimised results for $H, R s$ and $b$ are plotted in Figure 10(a).

\section{FE results and discussion}

\subsection{Uniaxial simulation results}

A series of fatigue tests were simulated using the software ABAQUS with a cubic geometry, under the same uniaxial cyclic loading condition. The objective of the fatigue test simulation is to check the validity of the material constants obtained in the optimisation procedure, and the validity and capability of the damage term in the model. Only one element is used in the study and the element type is 3D continuum eight-node brick element with full integration (C3D8 in ABAQUS).

For the strain range of $\pm 0.5 \%$, the stress-strain curves obtained from the FE simulation at cycles 1,300 and 600 are compared to the experimental results in Figure 10(b). The figure shows that the FE simulation is able to capture the main features of the cyclic plasticity behaviour, such as the size change and shift of the centres of elastic domain, the hysteresis loop, as well as the degradation of Young's modulus. The maximum stress evolutions in both FE simulation and experiment are plotted in Figure 11(a), as well as the damage evolution from both FE simulation and experimental postprocessing results. They show good prediction of the full life behaviour, including initial fast softening, steady linear softening, as well as the damage propagation.

For the strain ranges of $\pm 0.4 \%, \pm 0.25 \%$ and $\pm 0.2 \%$, the evolution of maximum stress $\sigma_{\max }$ against cycle number $N$ are plotted in Figure 11(b). They give good approximations of the peak stress evolution, especially after the initial fast softening period.

The stress-strain curves predicted by CDM were based on the assumption that microcrack sizes are smaller than the RVE size, so that damage is assumed to be homogeneous. However, when the microcrack size increases, the damage parameter would be greater than the critical damage value, and the CDM approach would be invalid. The fracture mechanics method, which deals with surface cracks, is a complementary method to the continuum damage approach.

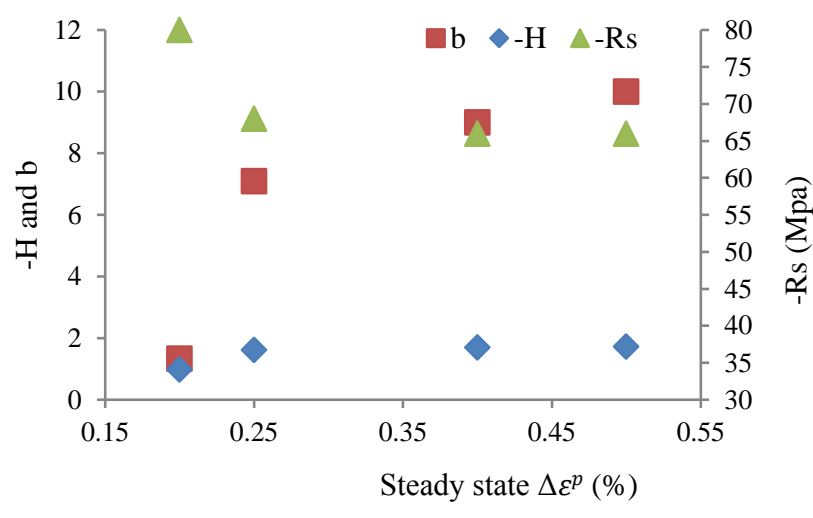

(b)

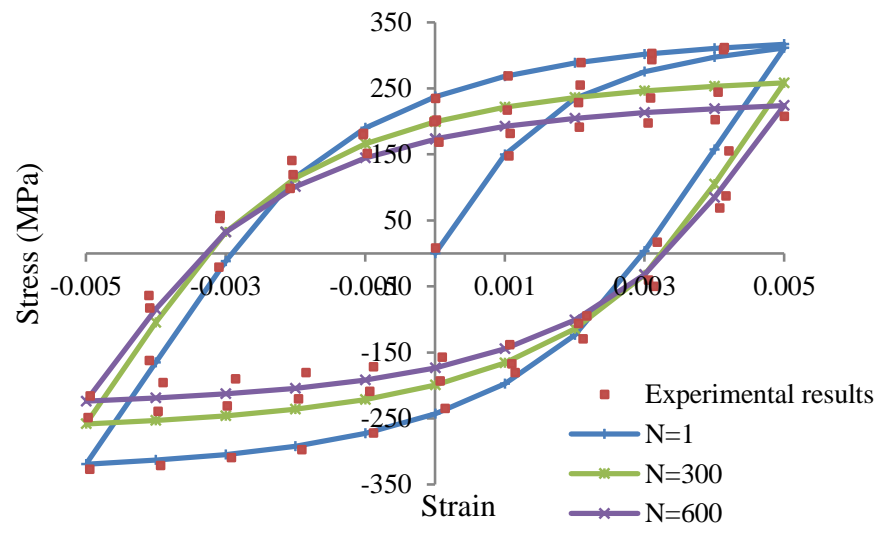

(a)

Figure 10. (a) Steady state plastic strain range $\Delta \varepsilon^{p}$ dependency of the material constants of isotropic hardening; (b) Experiment and FE simulation of stress-strain curve at cycle number 1, 300 and 600 for the strain range of $\pm 0.5 \%$ 
(a)

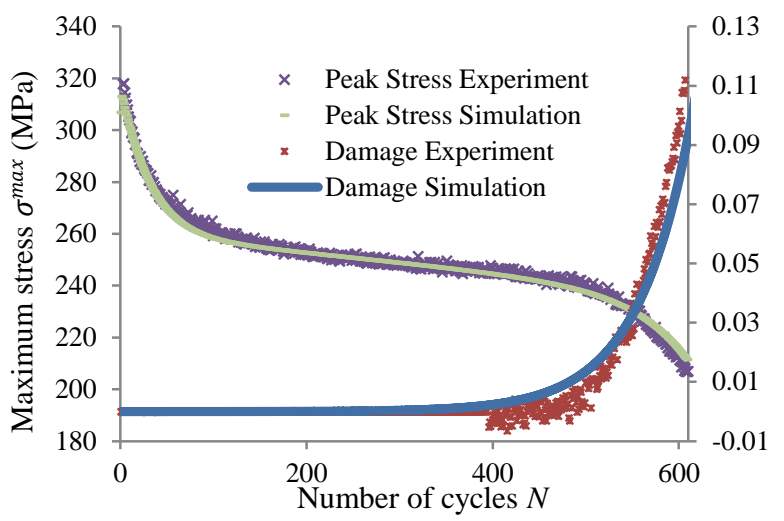

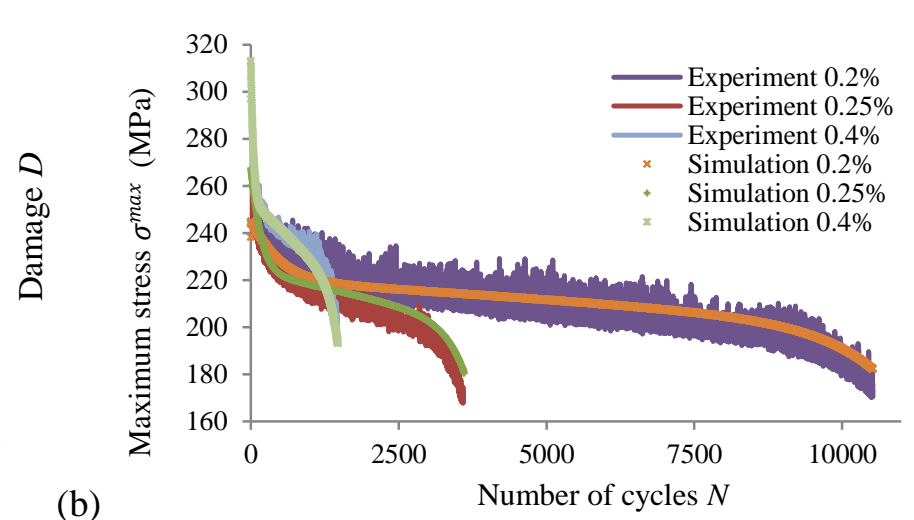

(b)

Figure 11. (a) Experiment and FE simulation of the maximum stress $\sigma^{\max }$ and damage $D$ evolution against cycle number for the strain range of $\pm 0.5 \%$; (b) Experiment and FE simulation of the maximum stress $\sigma^{\text {max }}$ against cycle number for the strain range of $\pm 0.4 \%, \pm 0.25 \%$ and $\pm 0.2 \%$

\subsection{Notched-bar simulation results}

In order to demonstrate and validate the multiaxial capability of the Chaboche visco-plasticity model, a notch-bar isothermal fatigue test was conducted under a fully reversed triangular waveform load with the maximum and the minimum average axial stresses of $\pm 300 \mathrm{MPa}$ on the minimum notch cross-section, cycle period of 20 seconds, and temperature of $600^{\circ} \mathrm{C}$. The specimen geometry and dimensions are shown in Figure 12(a). An extensometer with a gauge length of $12.5 \mathrm{~mm}$ was positioned symmetrically to the notch position. The direct output from the experiment was the relative displacement from the end of the extensometer to the notch root. The maximum and minimum values of the displacement during a cycle are denoted as $U_{\max }$ and $U_{\min }$. The experiment stops when there is a $5 \%$ increase of the displacement range $U_{\max }-U_{\min }$ of the extensometer from the projected straight line locus, at the cycle number 1238.

The FE simulation is applied to an axisymmetric notched-bar model. The element type used was CAX8R (8-node axisymmetric element with reduced integration, quadratic order) in ABAQUS. The experimental and FE simulation results for the maximum and minimum displacements during a cycle are plotted against the cycle number in Figure 12(b). The displacement reaches the same value at FE cycle number 1003. This difference is reasonable, since fatigue data are rather scattered in nature, and the predicted value of $N_{f}$ comes from the logarithmic Coffin-Mansion relationship.

The stress, strain and damage parameter distribution over the influential part of the results are presented in Figure 13. Seen from the end of FE loading cycle 1003, stress concentrates near the root with the maximum von Mises stress being $288 \mathrm{MPa}$ and the maximum in-plane stress of $420 \mathrm{MPa}$ (not shown here). However, the strain concentrates at the root up to $0.964 \%$, with a large amount of plasticity. In the rest of the specimen, little plastic behaviour was observed (strain range $<0.2 \%$ ). The damage parameter is also quite concentrated at the notch position up to 0.7 , which is already greater than the critical damage parameter $D_{c}$, with no obvious damage propagation around the notch. Since there is a large stress, strain and damage gradient in the element at the notch root, refining the mesh could improve the accuracy of the results. Further damage analysis may be conducted using a crack propagation analysis based on fracture mechanics. 
(a)

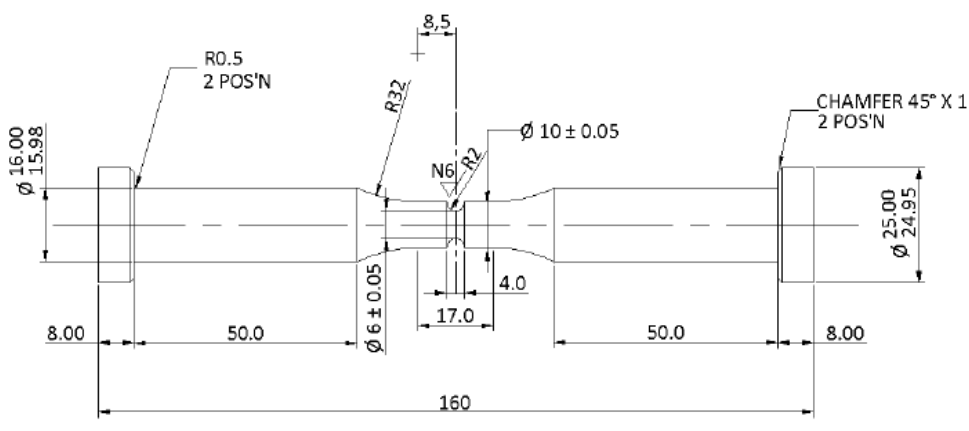

(b)

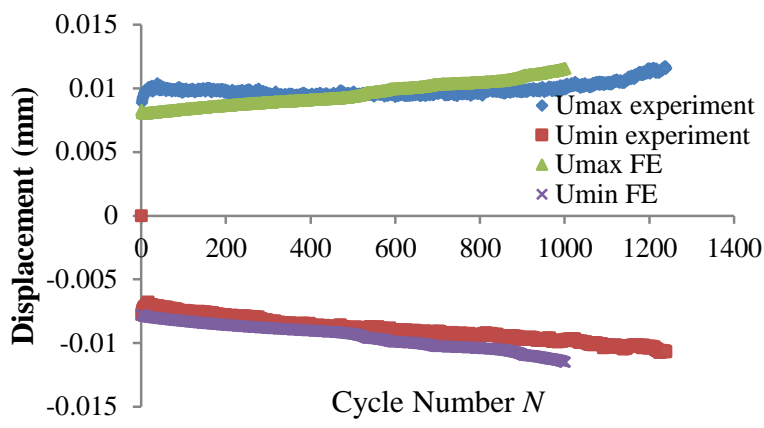

Figure 12.(a) The notched thermo-mechanical fatigue specimen used in the load-controlled test (units in $m m$ ); (b) The maximum $U_{\max }$ and minimum $U_{\min }$ relative displacement of the end of the extensometer to the root of the notch

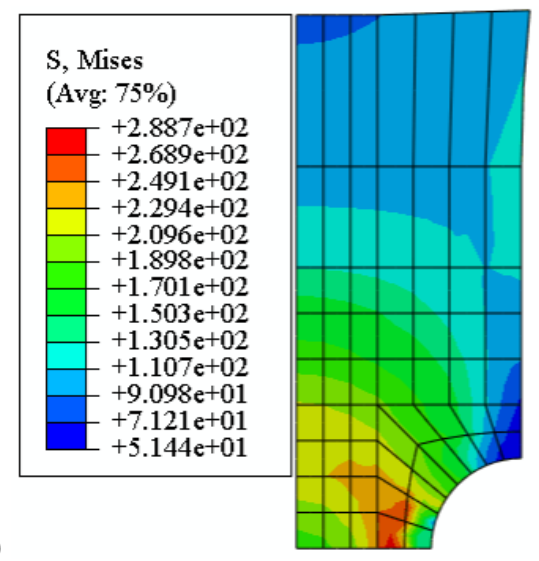

(a)

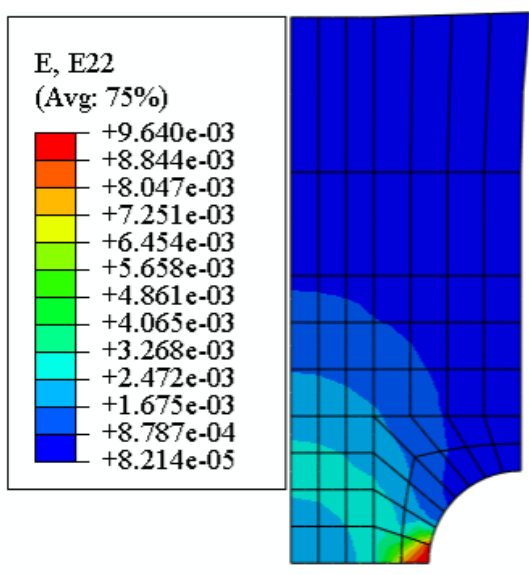

(c)

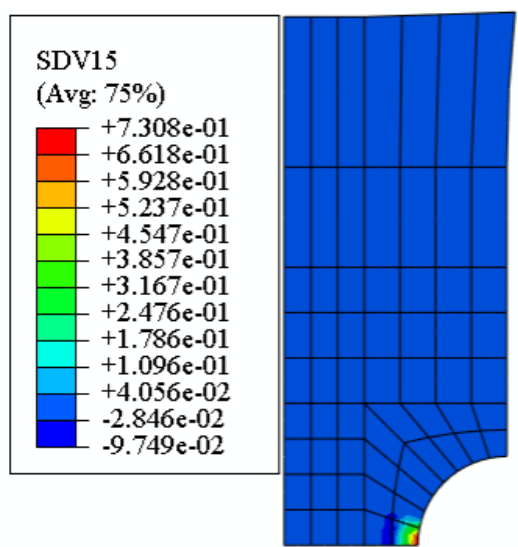

Figure 13. The output distribution at the end of loading of the cycle 1003: (a) von Mises stress (MPa) distribution over full geometry of the test specimen; (b) Strain in the loading direction over full geometry of the test specimen; (c) Damage parameter over the notch geometry of the test specimen

\section{Conclusions and future work}

A damage variable is coupled to the constitutive equations of the Chaboche elasto-visco-plastic model to describe the full life cyclic plasticity behaviour of a P91 steel at the temperature of $600{ }^{\circ} \mathrm{C}$ under low cycle fatigue conditions. A stress partition method is introduced to understand the fatigue softening behaviour of P91 at the high temperature of $600{ }^{\circ} \mathrm{C}$, and to determine an initial estimate of the material constants in the Chaboche visco-plasticity model. Further optimisation procedures with plastic strain range dependency of the material constants are introduced in the optimisation procedure in order to accurately predict the material behaviour, especially in the failure stage. Good approximations are achieved for the maximum stress evolution, especially in the linear softening stage and the damage propagation stage. The multiaxial capability of the ABAQUS UMAT coding is developed and is partially validated using the results from a notch bar test, from which it can be seen that reasonably accurate results have been obtained.

The major drawback of the current model is that no damage initiation criterion is included, and the damage model is highly dependent upon the accurate prediction of the number of cycles to failure $N_{f}$. The Manson-Coffin relationship is used in this case without considering the temperature and strain rate dependencies. Future work may involve using an acoustic system to record the state when damage initiates, to investigate the damage initiation criterion for P91 at high temperature. In addition, temperature dependency should be introduced to be applicable in a real component simulation. 


\section{References}

1. Bodner, S.R. and Y. Partom, Constitutive Equations for Elastic-Viscoplastic Strain-Hardening Materials. Journal of Applied Mechanics, 1975. 42 p. 385-389.

2. Chaboche, J.L., Continuum Damage Mechanics: Part I---General Concepts. Journal of Applied Mechanics, 1988. 55(1): p. 59-64.

3. Chaboche, J.L. and G. Rousselier, On the Plastic and Viscoplastic Constitutive Equations-Part I: Rules Developed With Internal Variable Concept. Journal of Pressure Vessel Technology, 1983. 105(2): p. 159-164.

4. Chaboche, J.L., Constitutive equations for cyclic plasticity and cyclic viscoplasticity. International Journal of Plasticity, 1989. 5(3): p. 247-302.

5. Ishmurzin, A., W. Ecker, M. Krobath, M. Orthaber, S. Marsoner, and T. Antretter, The cyclic elasto-viscoplastic behavior of a high-speed steel under forging conditions - experiments and simulations. Procedia Engineering, 2011. 10(0): p. 1991-1996.

6. Saad, A.A., W. Sun, T.H. Hyde, and D.W.J. Tanner, Cyclic softening behaviour of a P91 steel under low cycle fatigue at high temperature. Procedia Engineering, 2011. 10(0): p. 1103-1108.

7. Saad, A.A., Cyclic plasticity and creep of power plant materials, PhD thesis. 2012, University of Nottingham: Nottingham.

8. $\quad$ Kachanov, L.M., Rupture time under creep conditions. 1958.

9. Rabotnov, Y.N. Creep rupture 1968. Stanford University: Springer.

10. Lemaitre, J., A course on damage mechanics 2nd ed. 1996, Berlin: Springer.

11. Lemaitre, J. and R. Desmorat, Engineering damage mechanics : ductile, creep, fatigue and brittle failures 2004, Berlin ; London: Springer.

12. Wang, W., P. Buhl, and A. Klenk, A unified viscoplastic constitutive model with damage for multi-axial creepfatigue loading. International Journal of Damage Mechanics, 2014.

13. Zhang, G., Y. Zhao, F. Xue, J. Mei, Z. Wang, C. Zhou, and L. Zhang, Creep-fatigue interaction damage model and its application in modified 9Cr-1Mo steel. Nuclear Engineering and Design, 2011. 241(12): p. 4856-4861.

14. Lemaitre, J., Introduction to continuum damage mechanics, in Continuum damage mechanics of materials and structures O. Allix and F. Hild, Editors. 2002, Elsevier Science: Amsterdam ; London.

15. Lemaitre, J. and J.L. Chaboche, Mechanics of solid materials. 1994, Cambridge: Cambridge university press.

16. Chaboche, J.L. and F. Gallerneau, An overview of the damage approach of durability modelling at elevated temperature. Fatigue \& Fracture of Engineering Materials \& Structures, 2001. 24(6): p. 405-418.

17. Chaboche, J.L., A review of some plasticity and viscoplasticity constitutive theories. International Journal of Plasticity, 2008. 24(10): p. 1642-1693.

18. Powell, W.R., A note on yield curves in cyclic work softening. 1967, Brown University.

19. Ucak, A. and P. Tsopelas, Constitutive Model for Cyclic Response of Structural Steels with Yield Plateau. Journal of Structural Engineering, 2011. 137(2): p. 195-206.

20. Maciejewski, K. and H. Ghonem, Isotropic and Kinematic Hardening as Functions of Gamma Prime Precipitates in a Nickel-Based Superalloy. International Journal of Fatigue, (0).

21. Cottrell, A.H., Dislocations and plastic flow in crystals. 1953, UK: Oxford University Press.

22. Chaboche, J.L., On some modifications of kinematic hardening to improve the description of ratchetting effects. International Journal of Plasticity, 1991. 7(7): p. 661-678.

23. Frederick, C.O. and P.J. Armstrong, A mathematical representation of the multiaxial Bauschinger effect. Materials at High Temperatures, 2007. 24(1): p. 1-26.

24. Chan, K., A microstructure-based fatigue-crack-initiation model. Metallurgical and Materials Transactions A, 2003. 34(1): p. 43-58.

25. British Standard: Metallic materials-Fatigue testing-Strain-controlled thermomechanical fatigue testing method: BS ISO 12111:2011(E). 2011, BSI Standards Publication: UK.

26. Ma, A., F. Roters, and D. Raabe, A dislocation density based constitutive model for crystal plasticity FEM including geometrically necessary dislocations. Acta Materialia, 2006. 54(8): p. 2169-2179.

27. Norfleet, D.M., D.M. Dimiduk, S.J. Polasik, M.D. Uchic, and M.J. Mills, Dislocation structures and their relationship to strength in deformed nickel microcrystals. Acta Materialia, 2008. 56(13): p. 2988-3001.

28. Dunne, F. and N. Petrinic, Introduction to computational plasticity 2005: Oxford University Press.

29. Fournier, B., M. Sauzay, C. Caës, M. Noblecourt, and M. Mottot, Analysis of the hysteresis loops of a martensitic steel: Part I: Study of the influence of strain amplitude and temperature under pure fatigue loadings using an enhanced stress partitioning method. Materials Science and Engineering: A, 2006. 437(2): p. 183-196. 
30. Gorash, Y., H. Altenbach, and G. Lvov, Modelling of high-temperature inelastic behaviour of the austenitic steel AISI type 316 using a continuum damage mechanics approach. The Journal of Strain Analysis for Engineering Design, 2012. 47(4): p. 229-243.

31. Giroux, P.F., Experimental study and simulation of cyclic softening of tempered martensite ferritic steels. PhD thesis. 2011, Ecole Nationale Superieure des Mines de Paris 


\section{Appendix A. Flowchart of the implementation of the UMAT for the Chaboche visco- plasticity model}

Given $\boldsymbol{\sigma}_{n}, \boldsymbol{X}_{n}, R_{n}, p$ at time $t_{n}$, ABAQUS solver determines $\Delta t$ and $\Delta \boldsymbol{\varepsilon}$

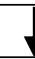

Elastic Predictor: Compute predicted elastic Jacobian matrix $\tilde{\boldsymbol{J}}^{\boldsymbol{e}}$,

$\boldsymbol{\sigma}_{n+1}=\boldsymbol{\sigma}_{n}+\tilde{\boldsymbol{J}}^{\boldsymbol{e}} d \boldsymbol{\varepsilon}, \boldsymbol{X}_{n+1}=\boldsymbol{X}_{n}, R_{n+1}=R_{n}, \Delta \mathrm{p}=0$

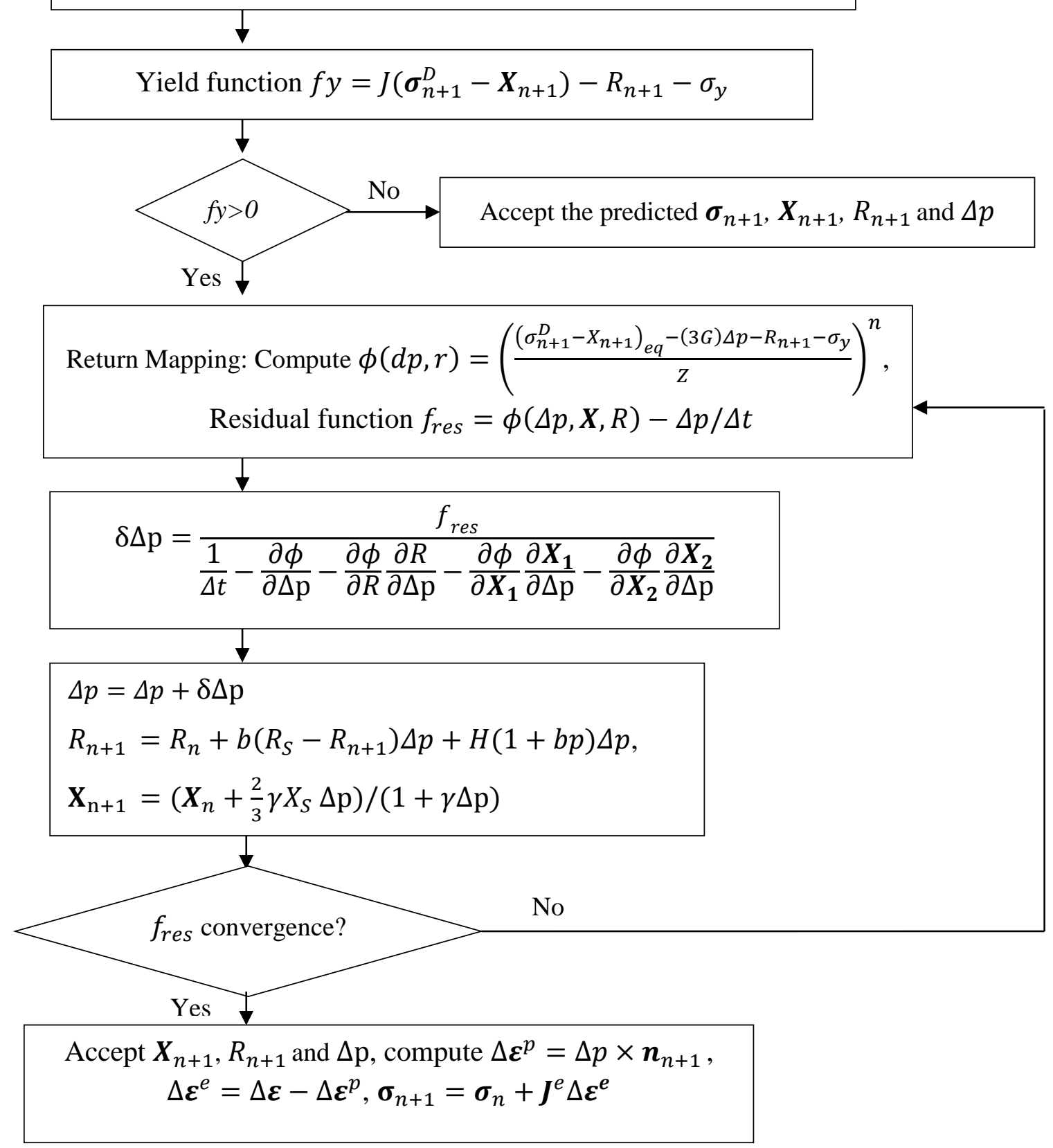




\section{Appendix B. Flowchart of the optimisation procedure in MATLAB}

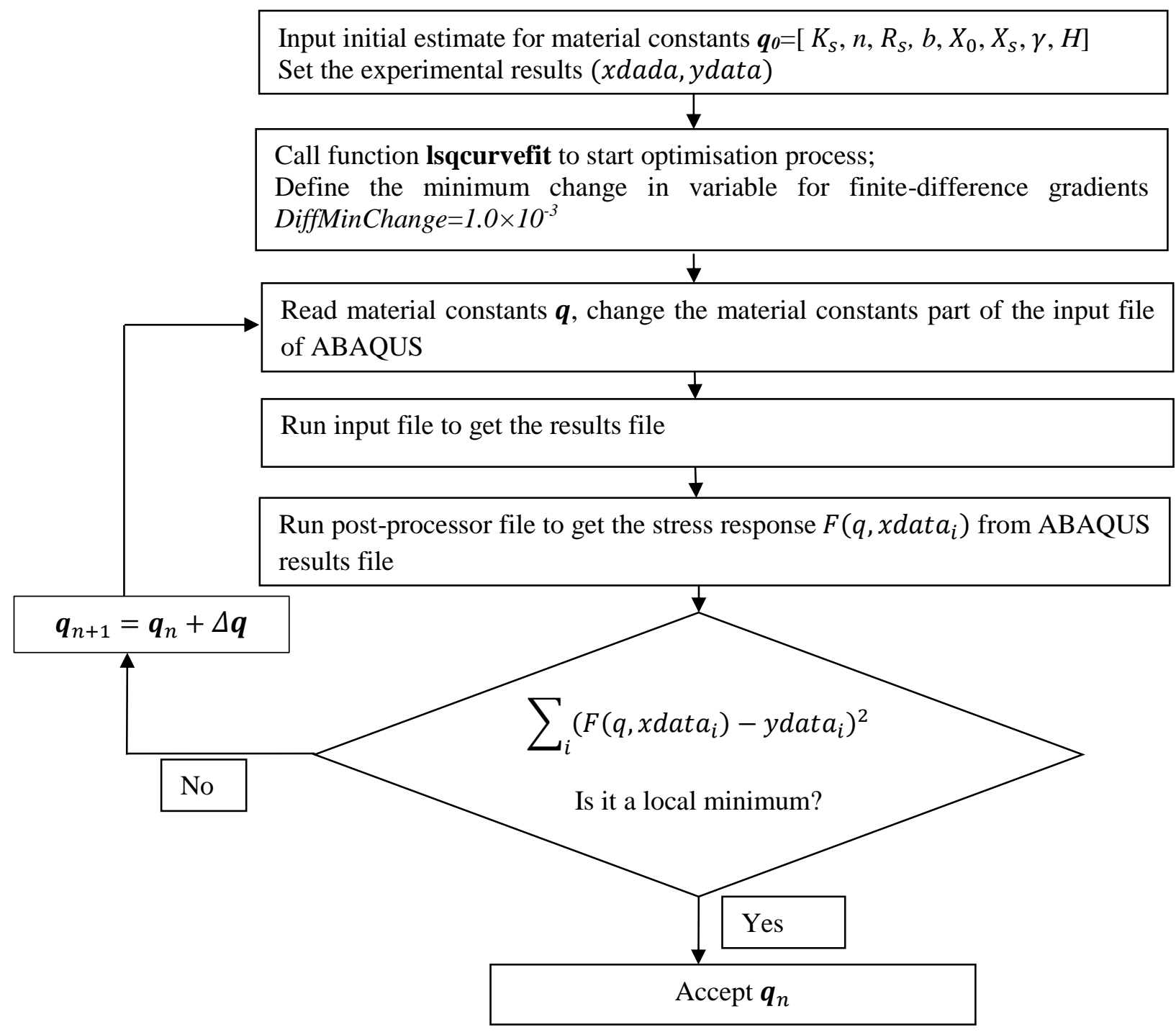

